\title{
Applications of uniform asymptotic regularity to fixed point theorems
}

\author{
Sławomir Borzdyński and Andrzej Wiśnicki
}

\begin{abstract}
In this paper, we show that there are no nontrivial surjective uniformly asymptotically regular mappings acting on a metric space, and we derive some consequences of this fact. In particular, we prove that a jointly continuous left amenable or left reversible semigroup generated by firmly nonexpansive mappings on a bounded $\tau$-compact subset of a Banach space has a common fixed point, and we give a qualitative complement to the Markov-Kakutani theorem.
\end{abstract}

Mathematics Subject Classification. Primary 47H10; Secondary 46B20, $47 \mathrm{H} 09$.

Keywords. Asymptotic regularity, fixed point, firmly nonexpansive mapping, averaged mapping, amenable semigroup, weak topologies, MarkovKakutani theorem, affine retraction.

\section{Introduction}

The notion of asymptotic regularity was introduced in 1966 by Browder and Petryshyn [6] in connection with the study of fixed points of nonexpansive mappings. Recall that a mapping $T: M \rightarrow M$ acting on a metric space $(M, d)$ is asymptotically regular if

$$
\lim _{n \rightarrow \infty} d\left(T^{n} x, T^{n+1} x\right)=0
$$

for all $x \in M$. Krasnoselskii [16] proved that if $K$ is a compact convex subset of a uniformly convex Banach space and if $T: K \rightarrow K$ is nonexpansive (i.e., $\|T x-T y\| \leq\|x-y\|$ for $x, y \in K$ ), then for any $x \in K$, the sequence $\left\{\left(\frac{1}{2} I+\frac{1}{2} T\right)^{n} x\right\}$ converges to a fixed point of $T$. He used the fact that the averaged mapping $\frac{1}{2}(I+T)$ is asymptotically regular. Subsequently, Ishikawa [15] proved that if $C$ is a bounded closed convex subset of a Banach space $E$ and $T: C \rightarrow C$ is nonexpansive, then the mapping $T_{\alpha}=(1-\alpha) I+\alpha T$ is asymptotically regular for each $\alpha \in(0,1)$. Independently, Edelstein and O'Brien [9] showed that $T_{\alpha}$ is uniformly asymptotically regular over $x \in C$, and Goebel 
and Kirk [10] proved that the convergence is uniform with respect to all nonexpansive mappings from $C$ into $C$. Other examples of asymptotically regular mappings are given by the result of Anzai and Ishikawa [1] - if $T$ is an affine mapping acting on a bounded closed convex subset of a locally convex space $X$, then $T_{\alpha}=(1-\alpha) I+\alpha T$ is uniformly asymptotically regular.

Another important class of nonlinear operators are firmly nonexpansive mappings, i.e., mappings with the property

$$
\|T x-T y\| \leq\|\alpha(x-y)+(1-\alpha)(T x-T y)\|
$$

for all $x, y \in C$ and $\alpha \in(0,1)$. Their origin is associated with the study of maximal monotone operators in Hilbert spaces. Reich and Shafrir [22] proved that every firmly nonexpansive mapping $T: C \rightarrow C$ is asymptotically regular provided $C$ is bounded. For a thorough treatment of firmly nonexpansive mappings we refer the reader to [2].

Quite recently, Bader, Gelander and Monod [3] proved a remarkable fixed point theorem for affine isometries in $L$-embedded Banach spaces preserving a bounded subset $A$ and showed its several applications. In particular, they simplified the Losert's proof and simultaneously obtained the optimal solution to the "derivation problem" studied since the 1960s. The present paper is partly motivated by the Bader-Gelander-Monod theorem. The paper [8] is also relevant at this point. Our basic observation is Proposition 2.2-there are no nontrivial surjective uniformly asymptotically regular mappings. It appears to be quite useful in fixed point theory of nonexpansive and affine mappings.

The well-known Day generalization of the Markov-Kakutani fixed point theorem asserts that a semigroup $S$ is left amenable if and only if, whenever $S$ acts as an affine continuous mapping on a nonempty compact convex subset $C$ of a Hausdorff locally convex space, $S$ has a common fixed point in $C$. In 1976, T.-M. Lau [17] posed the problem of characterizing left amenability of a semigroup $S$ in terms of the fixed point property for nonexpansive mappings. Consider the following fixed point property:

$\left(F_{*}\right)$ Whenever $\mathcal{S}=\left\{T_{s}: s \in S\right\}$ is a representation of $S$ as normnonexpansive mappings on a nonempty weak* compact convex set $C$ of a dual Banach space $E$ and the mapping $(s, x) \rightarrow T_{s}(x)$ from $S \times C$ to $C$ is jointly continuous, where $C$ is equipped with the weak ${ }^{*}$ topology of $E$, then there is a common fixed point for $\mathcal{S}$ in $C$.

It is not difficult to show (see, e.g., [19, p. 528]) that property $\left(F_{*}\right)$ implies that $S$ is left amenable. Whether the converse is true is still an open problem though a few partial results are known. Lau and Takahashi [18, Theorem 5.3] proved that the answer is affirmative if $C$ is separable. Recently, the authors of the present paper were able to prove that commutative semigroups have the $\left(F_{*}\right)$ property (see [5, Theorem 3.6]). We show in Section 3 that a jointly continuous left amenable or left reversible semigroup generated by firmly nonexpansive mappings acting on a bounded $\tau$-compact subset of a 
Banach space has a common fixed point. This is a partial result but the techniques developed in this paper may lead to a solution of the original problem too.

In Section 4 we apply our techniques to the case of commutative semigroups of affine mappings and give the qualitative complement to the MarkovKakutani theorem. In spite of its simplicity, the result seems to be generally new even for the weak topology, see Remark 4.6.

\section{Preliminaries}

Let $(M, d)$ be a metric space. In this paper, we focus on uniformly asymptotically regular (UAR) self-mappings on $M$, i.e., mappings $T: M \rightarrow M$ which satisfy the condition

$$
\lim _{n \rightarrow \infty} \sup _{x \in M} d\left(T^{n+1} x, T^{n} x\right)=0 .
$$

The following observation is our basis for using uniform asymptotic regularity to generate fixed points.

Lemma 2.1. A surjective mapping $T: M \rightarrow M$ with the property

$$
\inf _{n} \sup _{x \in M} d\left(T^{n+1} x, T^{n} x\right)=0
$$

is the identity mapping.

Proof. Take $\varepsilon>0$. The assured property gives us a positive integer $n$ such that

$$
\forall x \in M \quad d\left(T^{n+1} x, T^{n} x\right)<\varepsilon .
$$

Take arbitrary $y \in M$. From the surjectivity of $T$, there exists $x \in M$ such that $T^{n} x=y$. Then the above inequality turns out to be $d(y, T y)<\varepsilon$. Since $\varepsilon>0$ is arbitrary, we have $T y=y$ and consequently, $T=\mathrm{Id}$.

Proposition 2.2. There are no nontrivial surjective UAR mappings.

Notice that uniformity assumption is necessary as $T x=x^{2}$ defined on $[0,1]$ meets the demands of the above lemma, except it is merely asymptotically regular and obviously not the identity.

Let $C$ be a convex subset of a Banach space $X$ and $\alpha \in(0,1)$. For brevity and symmetry, we denote $1-\alpha$ by $\bar{\alpha}$. A mapping of the form

$$
T=\alpha I+\bar{\alpha} S
$$

is called ( $\alpha$-)averaged nonexpansive (resp., affine) provided that $S: C \rightarrow C$ is nonexpansive (resp., affine). The term "averaged mapping" was coined in [4]. Whenever a $\tau$-topology on $C$ is mentioned it is assumed to be Hausdorff. A set $C$ is called $T$-invariant if $T(C) \subset C$. 


\section{Applications to nonexpansive mappings}

We start by giving a few statements which result in the uniform asymptotic regularity. The first one was proved by Edelstein and O'Brien [9, Theorem 1] (see also [10, Theorem 2]).

Lemma 3.1. An averaged nonexpansive mapping $T: C \rightarrow C$ defined on a convex and bounded subset $C$ of a Banach space is UAR.

Recall that in a Hilbert space a mapping $T: C \rightarrow C$ is firmly nonexpansive if and only if $T=\frac{1}{2}(I+S)$ for a nonexpansive mapping $S$. In general, there is no such relation. However, a counterpart of Lemma 3.1 can be drawn for firmly nonexpansive mappings by combining a method of $[11$, Theorem 9.4] with [22, Theorem 1].

Lemma 3.2. A firmly nonexpansive mapping $T: C \rightarrow C$ defined on a bounded subset of a Banach space $E$ is UAR.

Proof. Let $C$ be a bounded subset of $E$ and put

$$
E^{\star}=\left\{S \in E^{C}:\|S\|<\infty\right\},
$$

where

$$
\|S\|=\sup \{\|S x\|: x \in C\}
$$

is the uniform norm. Let $C^{\star}=C^{C}$. It is known that $E^{\star}$ is a Banach space. Also, $C^{\star}$ inherits boundedness from $C$ :

$$
\forall \forall_{T \in C^{\star}} \quad \sup _{x \in C}\|T x\| \leq M \Longrightarrow \sup _{T \in C^{\star}}\|T\| \leq M .
$$

For $T \in C^{\star}$ define $\Psi_{T}: C^{\star} \rightarrow C^{\star}$ by

$$
\Psi_{T} S=T \circ S .
$$

If $T$ is firmly nonexpansive, then

$$
\begin{aligned}
\left\|\left(\Psi_{T} S-\Psi_{T} N\right) x\right\| & =\|T(S x)-T(N x)\| \\
& \leq\left\|\left(\alpha(S-N)+\bar{\alpha}\left(\Psi_{T} S-\Psi_{T} N\right)\right) x\right\|
\end{aligned}
$$

and, by taking suprema, we conclude that $\Psi_{T}$ is also firmly nonexpansive.

Now, from [22, Theorem 1] we have

$$
\lim _{n}\left\|\Psi_{T}^{n+1} S-\Psi_{T}^{n} S\right\|=\lim _{n}\left\|\frac{\Psi_{T}^{n} S}{n}\right\|=0,
$$

where the last equality follows from boundedness of $C^{\star}$. Taking $S$ as the identity on $C$, and unwinding definitions of $\|\cdot\|$ and $\Psi_{T}$, we get

$$
\lim _{n} \sup _{x \in C}\left\|T^{n+1} x-T^{n} x\right\|=0
$$

which is the desired conclusion.

Now from the ingredients above we have instantly the following result. 
Theorem 3.3. If for a firmly nonexpansive (resp., averaged nonexpansive) mapping $T: C \rightarrow C$ defined on a subset (resp., convex subset) of a Banach space there exists a nonempty bounded set $D \subset C$ such $T(D)=D$, then $D \subset \operatorname{Fix} T$. In particular, Fix $T$ is nonempty.

Corollary 3.4. Suppose that $C$ is a closed convex subset of a Banach space and $T: C \rightarrow C$ is nonexpansive. If for some $\alpha \in(0,1)$, the mapping $F=$ $(I-\alpha T)^{-1} \bar{\alpha} I$ is surjective on a (nonempty) bounded set $D \subset C$, then $T$ has a fixed point.

Proof. It follows from the fact that $F$ is firmly nonexpansive (see [11, p. 122]) and Fix $F=\operatorname{Fix} T$.

Below we use our technique to obtain fixed point theorems for semigroups of uniformly asymptotically regular mappings. But first let us introduce some notions.

Definition 3.5. We say that $\mathcal{S}=\left\{T_{s}: s \in S\right\}$ is a representation of a semigroup $S$ on a topological space $(C, \tau)$ if for each $s \in S, T_{s}$ is a mapping from $C$ into $C$ and $T_{s t} x=T_{s}\left(T_{t} x\right)$ for every $s, t \in S$ and $x \in C$.

Since, due to Lemma 2.1, surjectivity is intertwined into all results of this paper, the following definition seems natural.

Definition 3.6. We say that the representation $\mathcal{S}$ on the space $C$ is subsurjective if there is a (nonempty) set $D \subset C$ such that every $T \in \mathcal{S}$ is surjective on $D$.

There is a fundamental example of such representations.

Lemma 3.7. Every commuting semigroup of continuous mappings represented on a compact space is subsurjective on it.

Proof. Using the Kuratowski-Zorn lemma, we get a minimal compact $\mathcal{S}$ invariant set $D$. Now, picking any $T \in \mathcal{S}$, we notice that for every $P \in \mathcal{S}$,

$$
P(D) \subset D \Longrightarrow T P(D) \subset T(D) \Longrightarrow P T(D) \subset T(D),
$$

meaning that $T(D)$ is $\mathcal{S}$-invariant. It is also compact so, by the minimality of $D, T(D)=D$.

Let $S$ be a semitopological semigroup, i.e., a semigroup with a Hausdorff topology such that the mappings $S \ni s \rightarrow t s$ and $S \ni s \rightarrow s t$ are continuous for each $t \in S$. Notice that every semigroup can be equipped with the discrete topology and then it is called a discrete semigroup. The semigroup $S$ is said to be left reversible if any two closed right ideals of $S$ have a nonvoid intersection. In this case $(S, \leq)$ is a directed set with the relation $a \leq b$ if and only if

$$
\{a\} \cup \overline{a S} \supset\{b\} \cup \overline{b S} .
$$

Let $\ell^{\infty}(S)$ be the Banach space of bounded real-valued functions on $S$ with the supremum norm. For $s \in S$ and $f \in \ell^{\infty}(S)$, we define the element $l_{s} f$ in $\ell^{\infty}(S)$ by

$$
l_{s} f(t)=f(s t)
$$


for every $t \in S$. Denote by $C_{b}(S)$ the closed subalgebra of $\ell^{\infty}(S)$ consisting of continuous functions and let $\operatorname{LUC}(S)$ be the space of bounded left uniformly continuous functions on $S$, i.e., all $f \in C_{b}(S)$ such that the mapping

$$
S \ni s \rightarrow l_{s} f
$$

from $S$ to $C_{b}(S)$ is continuous when $C_{b}(S)$ has the sup norm topology. Note that if $S$ is a topological group, then $\operatorname{LUC}(S)$ is equivalently the space of bounded right uniformly continuous functions on $S$ (see [13]). A semigroup $S$ is called left amenable if there exists a left invariant mean on $\operatorname{LUC}(S)$, i.e., a functional $\mu \in \operatorname{LUC}(S)^{*}$ such that $\|\mu\|=\mu\left(I_{S}\right)=1$ and

$$
\mu\left(l_{s} f\right)=\mu(f)
$$

for each $s \in S$ and $f \in \operatorname{LUC}(S)$. If $S$ is discrete and left amenable, then $S$ is left reversible. In general, there is no such relation (see [14, p. 335]).

Let us introduce one more temporary definition.

Definition 3.8. If in the markings as stated in Definition $3.5, \mathcal{S}$ is a left reversible or left amenable semitopological semigroup and the mapping

$$
S \times C \ni(s, x) \rightarrow T_{s} x
$$

is jointly continuous, then we say that the semigroup is $(\tau$-)properly represented.

Since it is known that, notably, commuting semigroups are left amenable, we can now conveniently state a generalization of Lemma 3.7.

Lemma 3.9. Semigroups of mappings properly represented on a compact space are subsurjective.

Proof. Just use [20, Lemma 3.4] in the case of left reversible semigroups and [18, Lemma 5.1] for left amenable ones. Note also that in both cases the resulting set is compact.

Now, after we presented some decent examples of subsurjectivity, we formulate the main result of this section.

Theorem 3.10. Suppose that $(M, d)$ is a metric space. Then a subsurjective semigroup $\mathcal{S}$ generated by UAR mappings on $M$ has a common fixed point.

Proof. It follows from subsurjectivity that there exists $D \subset M$ such that $T_{s}(D)=D$ for $s \in S$. Now, Proposition 2.2 yields that every generator of $\mathcal{S}$ is the identity on $D$. It follows that $T_{s} x=x$ for every $x \in D$ and $s \in S$.

Combining the above theorem with Lemma 3.2 we obtain the following corollary.

Corollary 3.11. Let $\mathcal{S}$ be a subsurjective semigroup on a bounded subset $C$ of a Banach space whose generators consist of firmly nonexpansive mappings. Then $\mathcal{S}$ has a common fixed point. 
Note that a special case of the above corollary gives a partial answer to the problem of T.-M. Lau [20, Question 1] (as described in the Introduction) for semigroups generated by firmly nonexpansive mappings.

Corollary 3.12. Let $\mathcal{S}=\left\{T_{s}: s \in S\right\}$ be a representation of a left amenable semigroup on $w^{*}$-compact subset $C$ of a Banach space generated by firmly nonexpansive mappings such the mapping $S \times C \ni(s, x) \rightarrow T_{s} x$ is jointly continuous (where $C$ is equipped with the weak* topology). Then $\mathcal{S}$ has a common fixed point.

Assuming proper representability instead of just subsurjectivity, we get a result stronger than Theorem 3.10 .

Lemma 3.13. Let $\mathcal{S}$ be a properly represented semigroup generated by UAR mappings defined on a metric space $M$. Then every compact $\mathcal{S}$-invariant subset of $M$ contains a fixed point of $\mathcal{S}$.

\section{Applications to affine mappings}

Recall that a mapping $T: C \rightarrow C$ is said to be affine if

$$
T(\alpha x+\beta y)=\alpha T x+\beta T y
$$

for every $x, y \in C$ and $\alpha, \beta \geq 0, \alpha+\beta=1$.

The famous Markov-Kakutani theorem states that if $C$ is a nonempty compact and convex subset of a (Hausdorff) linear topological space $X$ and $\left\{T_{i}\right\}_{i \in I}$ a commutative family of continuous affine mappings of $C$ into $C$, then there exists a common fixed point $x \in C: T_{i} x=x$ for every $i \in I$. Furthermore, $\bigcap_{i \in I}$ Fix $T_{i}$ is compact and convex, and thus is an absolute retract provided $C$ is a metrizable subset of a locally convex space (see, e.g., [12, Theorem 7.7.5]). In particular, then, there exists a (continuous) retraction

$$
r: C \rightarrow \bigcap_{i \in I} \operatorname{Fix} T_{i}
$$

A natural question arises whether we can also obtain an affine retraction onto $\bigcap_{i \in I}$ Fix $T_{i}$. To the best of our knowledge, only very partial results regarding almost periodic actions are in the literature (see [21]). In this section we prove a general result of this kind. To this end we need the following lemma, see [1, Lemma 1] and [23, Theorem 3].

Lemma 4.1. An averaged affine self-map defined on a convex and bounded subset of a Banach space is UAR.

Proof. Let $T: C \rightarrow C$ be an $\alpha$-averaged mapping of an affine mapping $S$. Notice that from the affinity we get a quite compact binomial expansion:

$$
T^{n}=(\alpha I+\bar{\alpha} S)^{n}=\sum_{k=0}^{n}\left(\begin{array}{l}
n \\
k
\end{array}\right) \alpha^{(n-k)} \bar{\alpha}^{k} S^{k} .
$$


With the notation $\left(\begin{array}{c}n \\ n+1\end{array}\right)=\left(\begin{array}{c}n \\ -1\end{array}\right)=0$, we have

$$
\begin{aligned}
T\left(T^{n}\right) & =\alpha T^{n}+\sum_{k=0}^{n}\left(\begin{array}{l}
n \\
k
\end{array}\right) \alpha^{(n-k)} \bar{\alpha}^{k+1} S^{k+1} \\
& =\alpha T^{n}+\sum_{k=0}^{n+1}\left(\begin{array}{c}
n \\
k-1
\end{array}\right) \alpha^{(n-k+1)} \bar{\alpha}^{k} S^{k}
\end{aligned}
$$

Then

$$
T^{n}-T^{n+1}=\bar{\alpha} T^{n}-\sum_{k=0}^{n+1}\left(\begin{array}{c}
n \\
k-1
\end{array}\right) \alpha^{(n-k+1)} \bar{\alpha}^{k} S^{k}=\sum_{k=0}^{n+1}\left[\begin{array}{l}
n \\
k
\end{array}\right] S^{k},
$$

where

$$
\left[\begin{array}{l}
n \\
k
\end{array}\right]=\left(\begin{array}{l}
n \\
k
\end{array}\right) \alpha^{(n-k)} \bar{\alpha}^{k+1}-\left(\begin{array}{c}
n \\
k-1
\end{array}\right) \alpha^{(n-k+1)} \bar{\alpha}^{k} .
$$

We get that $\left[\begin{array}{l}n \\ k\end{array}\right]$ is nonnegative up to $m=\lfloor\bar{\alpha}(n+1)\rfloor$. Using the identity

$$
\left[\begin{array}{l}
n \\
k
\end{array}\right]+\left[\begin{array}{c}
n \\
k+1
\end{array}\right]=-\left(\begin{array}{c}
n \\
k-1
\end{array}\right) \alpha^{(n-k+1)} \bar{\alpha}^{k}+\left(\begin{array}{c}
n \\
k+1
\end{array}\right) \alpha^{(n-k+1)} \bar{\alpha}^{k+2}
$$

and having in mind boundary cases, we get by telescoping,

$$
\sum_{k=0}^{m}\left[\begin{array}{l}
n \\
k
\end{array}\right]=\left(\begin{array}{l}
n \\
m
\end{array}\right) \alpha^{(n-m)} \bar{\alpha}^{m+1}=a_{n} \geq 0
$$

Also, since our coefficients are negative above $m$, we similarly get

$$
\sum_{k=m+1}^{n+1}\left|\left[\begin{array}{l}
n \\
k
\end{array}\right]\right|=\left|\sum_{k=m+1}^{n+1}\left[\begin{array}{l}
n \\
k
\end{array}\right]\right|=\left|-\left(\begin{array}{l}
n \\
m
\end{array}\right) \alpha^{(n-m)} \bar{\alpha}^{m+1}\right|=a_{n} .
$$

Since $C$ is convex, it follows that for each $x \in C$,

$$
\begin{array}{r}
\sum_{k=0}^{m} \frac{\left[\begin{array}{l}
n \\
k
\end{array}\right]}{a_{n}} S^{k} x=x_{1} \in C, \\
\sum_{k=m+1}^{n+1} \frac{\left|\left[\begin{array}{l}
n \\
k
\end{array}\right]\right|}{a_{n}} S^{k} x=x_{2} \in C .
\end{array}
$$

Notice that

$$
\left\|T^{n} x-T^{n+1} x\right\|=a_{n}\left\|x_{1}-x_{2}\right\| \leq a_{n} \operatorname{diam} C .
$$

If $\alpha=\frac{1}{2}$ and $n$ is even, we have from the Stirling's approximation of the central binomial coefficient,

$$
a_{n}=\frac{1}{2^{n+1}}\left(\begin{array}{c}
n \\
\frac{1}{2} n
\end{array}\right) \approx \frac{1}{2^{n+1}} \frac{2^{n}}{\sqrt{\pi \frac{n}{2}}} \rightarrow 0 .
$$

The odd case, $n=2 k-1$ gives

$$
a_{n}=\frac{1}{2^{n}} \frac{(2 k-1) !}{k !(k-1) !}=\frac{1}{2^{n}} \frac{2 k}{2 k} \frac{(2 k-1) !}{k !(k-1) !}=\frac{1}{2^{n+1}} \frac{(2 k) !}{(k !)^{2}} \approx \frac{1}{2^{2 k}} \frac{2^{2 k}}{\sqrt{\pi k}} \rightarrow 0 .
$$


This proves that $a_{n}$ vanishes at infinity, and since $C$ is bounded and $a_{n}$ does not depend on $x$, we conclude that $T$ is UAR for $\alpha=\frac{1}{2}$ (which turns out to be sufficient for our applications). The case $\alpha>\frac{1}{2}$ can also be easily proved: define $U: C \rightarrow C$ as $\lambda$-averaged mapping of $S$ with $\lambda=2 \alpha$. Notice that $U$ is an affine mapping and $T$ is its $1 / 2$-averaged mapping, so once again $T$ is UAR. As for the case $\alpha>\frac{1}{2}$, we recommend the reader to compare [1, Lemma 1] or [23, Theorem 3].

Notice that in Lemma $3.13 \mathrm{D}$ need not be a convex set. This helps us to prove our final theorem. But first recall the following variant of Bruck's theorem (see [7, Theorem 3]).

Theorem 4.2. Let $(C, \tau)$ be a topological space and $\mathcal{S}$ a semigroup of mappings on $C$. Suppose that $\mathcal{S}$ is compact in the topology of pointwise convergence and each nonempty closed $\mathcal{S}$-invariant subset of $C$ contains a fixed point of $\mathcal{S}$. Then there exists in $\mathcal{S}$ a surjective mapping $r: C \rightarrow \operatorname{Fix} \mathcal{S}$ which is an idempotent: $r \circ r=r$.

The following corollary will be sufficient for us.

Corollary 4.3. Let $(C, \tau)$ be a compact topological space and $\mathcal{S}$ a semigroup of $\tau$-continuous affine mappings on $C$. Suppose that each nonempty closed $\mathcal{S}$ invariant subset of $C$ contains a fixed point of $\mathcal{S}$. Then there exists an affine and surjective idempotent mapping $r: C \rightarrow \operatorname{Fix} \mathcal{S}$.

Proof. Let $\overline{\mathcal{S}}$ denote the closure of $\mathcal{S}$ in $C^{C}$ in the $\tau$-product topology (i.e., topology of pointwise convergence with respect to the $\tau$-topology). Notice that if $S, T \in \overline{\mathcal{S}}$, then there exists nets $\left\{S_{\alpha}\right\}_{\alpha \in A},\left\{T_{\beta}\right\}_{\beta \in B}$ of mappings in $\mathcal{S}$ such that $S x=\tau$ - $\lim _{\alpha} S_{\alpha} x$ and $T x=\tau-\lim _{\beta} T_{\beta} x$ for $x \in C$. Notice that for every $\alpha,\left\{S_{\alpha} T_{\beta}\right\}_{\beta} \subset \mathcal{S}$ and from $\tau$-continuity of $S_{\alpha}$,

$$
\overline{\mathcal{S}} \ni \tau-\lim _{\beta}\left(S_{\alpha} T_{\beta}\right)=S_{\alpha}\left(\tau-\lim _{\beta} T_{\beta}\right)=S_{\alpha} T,
$$

where the above limits are to be understood in the topology of $\tau$-pointwise convergence. Hence $\left\{S_{\alpha} T\right\}_{\alpha} \subset \overline{\mathcal{S}}$ and again,

$$
\overline{\mathcal{S}}=\overline{\overline{\mathcal{S}}} \ni \tau-\lim _{\alpha} S_{\alpha} T=S \circ T
$$

which follows from the pointwise definition of the above limit. Thus $\overline{\mathcal{S}}$ is a semigroup. Since it is also compact as the closed subset of the compact set $C^{C}$ (in the $\tau$-product topology) and consists of affine mappings, we can apply Bruck's theorem to get in $\overline{\mathcal{S}}$ an affine idempotent $r$ from $C$ onto Fix $\overline{\mathcal{S}}$ which is our desired mapping since $\operatorname{Fix} \overline{\mathcal{S}}=\operatorname{Fix} \mathcal{S}$.

Theorem 4.4. Suppose that $\mathcal{S}$ is a $\tau$-properly represented semigroup generated by averaged affine mappings on $C$, where $C$ is a convex and $\tau$-compact bounded subset of a Banach space. Then there exists an affine, surjective idempotent $r: C \rightarrow \operatorname{Fix} \mathcal{S}$. If, moreover, elements of $\mathcal{S}$ are locally $\tau$-equicontinuous, then we can pick $r$ to be also $\tau$-continuous. 
Proof. Since from Lemma 4.1 generators of $\mathcal{S}$ are UAR, the existence of an affine, surjective idempotent $r$ follows instantly from Lemma 3.13 and Corollary 4.3.

Assume now that mappings from $\mathcal{S}$ are locally $\tau$-equicontinuous. Pick $x_{0} \in C$, a net $\left\{T_{\alpha}\right\}_{\alpha \in A} \subset \mathcal{S}$ and let $T$ be the limit of this net in the $\tau$-product topology. As earlier, let $\bar{A}$ denote the closure of $A$ in this topology. Pick any $B, U \in \tau_{0}$ such that $\bar{B} \subset U$, where $\tau_{0}$ denotes $\tau$-neighbourhoods of the origin. Then we conclude from local $\tau$-equicontinuity that

$$
\exists_{V \in \tau_{x_{0}}} \forall_{\alpha \in A} \forall_{x, y \in V} \quad T_{\alpha} x-T_{\alpha} y \in B,
$$

where $\tau_{x_{0}}$ denotes $\tau$-neighbourhoods of $x_{0}$. With $V$ satisfying the above condition, we have $T x-T y \in \bar{B} \subset U$. Thus

$$
\forall_{U \in \tau_{0}} \exists_{V \in \tau_{x_{0}}} \forall_{x, y \in V} \quad T x-T y \in U
$$

which means that each $T \in \overline{\mathcal{S}}$ is $\tau$-continuous at any $x_{0} \in C$. In particular, it relates to $r$.

We are now ready to state the following qualitative version of the Markov-Kakutani theorem.

Corollary 4.5. Suppose that $\mathcal{S}$ is a commutative family of $\tau$-continuous affine mappings on $C$, where $C$ is a convex and $\tau$-compact bounded subset of a $B a$ nach space. Then there exists an affine, surjective idempotent

$$
r: C \rightarrow \operatorname{Fix} \mathcal{S} \text {. }
$$

If, moreover, elements of $\mathcal{S}$ are locally $\tau$-equicontinuous, then there exists a $(\tau$-continuous) retraction onto $\operatorname{Fix} \mathcal{S}$.

Proof. Construct from $\mathcal{S}$ a semigroup $\hat{\mathcal{S}}$ generated by averaged mappings $\left\{\frac{1}{2} I+\frac{1}{2} T: T \in \mathcal{S}\right\}$ which is obviously commutative and consists of affine $\tau$ continuous mappings. Recall that a commutative semigroup is subsurjective on compact sets. Now it is enough to notice that $\hat{\mathcal{S}}$ is locally $\tau$-equicontinuous whenever $\mathcal{S}$ is, and apply Theorem 4.4 .

Remark 4.6. Note that in the case of $w$-topology the assumption about local $w$-equicontinuity can be changed to (strong) local equicontinuity because every strongly continuous affine mapping is weakly continuous. Even in this case the result seems to have been known only in strictly convex Banach spaces (see [21, Theorem 5.8]).

Remark 4.7. The results of this section are formulated for subsets of Banach spaces only, but similar arguments apply to the case of locally convex spaces, as well.

Note, on the margin, that starting again from Lemma 3.13 and basically repeating the scheme presented in this section, we get a "firmly nonexpansive" counterpart of Theorem 4.4. 
Theorem 4.8. Suppose that $\mathcal{S}$ is a $\tau$-properly represented semigroup generated by firmly nonexpansive mappings on $C$, where $C$ is a $\tau$-compact bounded subset of a Banach space. Then there exists in $\overline{\mathcal{S}}$ an idempotent mapping $r$ from $C$ onto Fix $\mathcal{S}$. If, moreover, the norm is $\tau$-lower semicontinuous, then $\operatorname{Fix} \mathcal{S}$ is a nonexpansive retract of $C$.

Furthermore, we have the following theorem.

Theorem 4.9. Suppose that $\mathcal{S}$ is a properly represented semigroup generated by UAR mappings on a compact metric space $M$. Then there exists in $\overline{\mathcal{S}}$ a surjective idempotent $r: M \rightarrow \operatorname{Fix} \mathcal{S}$.

\section{References}

[1] K. Anzai and S. Ishikawa, On common fixed points for several continuous affine mappings. Pacific J. Math. 72 (1977), 1-4.

[2] D. Ariza-Ruiz, G. López-Acedo and V. Martín-Márquez, Firmly nonexpansive mappings. J. Nonlinear Convex Anal. 15 (2014), 61-87.

[3] U. Bader, T. Gelander and N. Monod, A fixed point theorem for $L_{1}$ spaces. Invent. Math. 189 (2012), 143-148.

[4] J. B. Baillon, R. E. Bruck and S. Reich, On the asymptotic behavior of nonexpansive mappings and semigroups in Banach spaces. Houston J. Math. 4 (1978), 1-9.

[5] S. Borzdyński and A. Wiśnicki, A common fixed point theorem for a commuting family of weak* continuous nonexpansive mappings. Studia Math. 225 (2014), 173-181.

[6] F. E. Browder and W. V. Petryshyn, The solution by iteration of nonlinear functional equations in Banach spaces. Bull. Amer. Math. Soc. 72 (1966), 571575.

[7] R. E. Bruck, Jr., A common fixed point theorem for a commuting family of nonexpansive mappings. Pacific J. Math. 53 (1974), 59-71.

[8] R. E. Bruck and S. Reich, Nonexpansive projections and resolvents of accretive operators in Banach spaces. Houston J. Math. 3 (1977), 459-470.

[9] M. Edelstein and R. C. O'Brien, Nonexpansive mappings, asymptotic regularity and successive approximations. J. Lond. Math. Soc. (2) 17 (1978), 547-554.

[10] K. Goebel and W. A. Kirk, Iteration processes for nonexpansive mappings. In: Topological Methods in Nonlinear Functional Analysis, S. P. Singh, S. Thomeier, B. Watson (eds.), AMS, Providence, RI, 1983, 115-123.

[11] K. Goebel and W. A. Kirk, Topics in Metric Fixed Point Theory. Cambridge University Press, Cambridge, 1990.

[12] A. Granas and J. Dugundji, Fixed Point Theory. Springer-Verlag, New York, 2003.

[13] E. Hewitt and K. A. Ross, Abstract Harmonic Analysis. Vol. I: Structure of Topological Groups. Integration Theory, Group Representations. Academic Press, New York, 1963.

[14] R. D. Holmes and A. T. Lau, Non-expansive actions of topological semigroups and fixed points. J. Lond. Math. Soc. (2) 5 (1972), 330-336. 
[15] S. Ishikawa, Fixed points and iteration of a nonexpansive mapping in a Banach space. Proc. Amer. Math. Soc. 59 (1976), 65-71.

[16] M. A. Krasnoselskii, Two observations about the method of successive approximations. Uspehi Math. Nauk. 10 (1955), 123-127.

[17] A. T.-M. Lau, Some fixed point theorems and their applications to $W^{*}$-algebras. In: Fixed Point Theory and Applications, S. Swaminathan (ed.), Academic Press, New York, 1976, 121-129.

[18] A. T.-M. Lau and W. Takahashi, Invariant means and fixed point properties for non-expansive representations of topological semigroups. Topol. Methods Nonlinear Anal. 5 (1995), 39-57.

[19] A. T.-M. Lau and W. Takahashi, Fixed point and non-linear ergodic theorems for semigroups of non-linear mappings. In: Handbook of Metric Fixed Point Theory, W. A. Kirk, B. Sims (eds.), Kluwer Academic Publishers, Dordrecht, 2001, 515-553.

[20] A. T.-M. Lau and Y. Zhang, Fixed point properties for semigroups of nonlinear mappings and amenability. J. Funct. Anal. 263 (2012), 2949-2977.

[21] V. Q. Phóng, Nonlinear almost periodic actions of semigroups. In: Functional Analysis, K. D. Bierstedt at al. (eds.), Marcel Dekker, 1994, 71-94.

[22] S. Reich and I. Shafrir, The asymptotic behavior of firmly nonexpansive mappings. Proc. Amer. Math. Soc. 101 (1987), 246-250.

[23] H. K. Xu and I. Yamada, Asymptotic regularity of linear power bounded operators. Taiwanese J. Math. 10 (2006), 417-429.

Sławomir Borzdyński

Institute of Mathematics

Maria Curie-Skłodowska University

20-031 Lublin

Poland

e-mail: slawomir.borzdynski@gmail.com

Andrzej Wiśnicki

Department of Mathematics

Rzeszów University of Technology

Al. Powstańców Warszawy 12

35-959 Rzeszów

Poland

e-mail: awisnicki@prz.edu.pl

Open Access This article is distributed under the terms of the Creative Commons Attribution 4.0 International License (http://creativecommons.org/licenses/by/4.0/), which permits unrestricted use, distribution, and reproduction in any medium, provided you give appropriate credit to the original author(s) and the source, provide a link to the Creative Commons license, and indicate if changes were made. 